Pacific Journal of Mathematics

COMPACTNESS PROPERTIES OF ABSTRACT KERNEL
OPERATORS Charalambos D. Aliprantis,
OWen Sidney Burkinshaw and M. Duhoux 


\title{
COMPACTNESS PROPERTIES OF ABSTRACT KERNEL OPERATORS
}

\author{
C. D. Aliprantis, O. Burkinshaw, and M. Duhoux
}

Let $E$ and $F$ be two Banach function spaces on two $\sigma$ finite measure spaces $(Y, \Sigma, \nu)$ and $(X, S, \mu)$ respectively. Then an operator $T: E \rightarrow F$ is called a kernel operator, if there exists a $\mu \times \nu$-measurable real valued function $K(x, y)$ on $X \times Y$ such that for each $f \in E$ we have

$$
\begin{aligned}
T f(x)= & \int_{Y} K(x, y) f(y) d \nu(y) \text { for } \mu \text {-almost all } x, \text { and } \\
& \int_{Y}|K(\cdot, y) f(y)| d \nu(y) \in F .
\end{aligned}
$$

It is well known that every kernel operator belongs to the band $\left(E^{\prime} \otimes F\right)^{d d}$ generated by the finite rank operators, and under certain conditions $\left(E^{\prime} \otimes F\right)^{d d}$ consists precisely of all kernel operators.

In this paper we consider $E$ and $F$ to be two locally convex-solid Riesz spaces. Motivated by the above remarks, we call every operator in $\left(E^{\prime} \otimes F\right)^{d d}$ that can be written as a difference of two positive weakly continuous operators, an abstract kernel operator. We characterize the abstract kernel operators that map bounded sets onto precompact sets. In the process we generalize known characterizations of compact kernel operators and obtain some interesting new ones.

1. Preliminaries. Unless otherwise stated, all topological vector spaces encountered in this paper will be assumed to be Hausdorff. For notation and terminology concerning locally solid Riesz spaces not explained below, we refer the reader to [1]. For terminology concerning locally convex spaces we follow [14].

A linear topology $\tau$ on a Riesz space is said to be locally solid if $\tau$ has a neighborhood basis at zero consisting of solid sets. (A set $V$ is said to be solid whenever $|x| \leqq|y|$ and $y \in V$ imply $x \in V$.) If $\tau$ is both locally convex and locally solid, then it is referred to as a locally convex-solid topology, and in this case, $\tau$ has a basis at zero consisting of solid and convex sets. The main properties connecting topological and order continuity needed for this work are the following:

1. The Lebesgue property: $u_{\alpha} \downarrow 0$ implies $u_{\alpha} \stackrel{\tau}{\rightarrow} 0$.

2. The pre-Lebesgue property: $0 \leqq u_{n} \uparrow \leqq u$ implies that $\left\{u_{n}\right\}$ is $\tau$-Cauchy. 
The Lebesgue property implies the pre-Lebesgue property [1, $\mathrm{p}$. 66]. For topologically complete locally solid Riesz spaces the two concepts coincide [1, p. 66]. The pre-Lebesgue property is equivalent to the statement: Every disjoint order bounded sequence is $\tau$-convergent to zero; see [1, Th. 10.1, p. 64].

Let $(E, \tau)$ be a locally convex-solid Riesz space. The topological dual of $(E, \tau)$ will be denoted by $E^{\prime}$. The absolute weak topology $|\sigma|\left(E, E^{\prime}\right)$ is the locally convex-solid topology on $E$ generated by the family of Riesz seminorms $\left\{\rho_{f}: f \in E^{\prime}\right\}$, where $\rho_{f}(x)=|f|(|x|)$ for each $x \in E$. Similarly, $|\sigma|\left(E^{\prime}, E\right)$ is the locally convex-solid topology generated on $E^{\prime}$ by the family of Riesz seminorms $\left\{\rho_{x}: x \in E\right\}$, where $\rho_{x}(f)=|f|(|x|)$ for each $f \in E^{\prime}$. The topology $|\sigma|\left(E^{\prime}, E\right)$ is always a Lebesgue topology, while $|\sigma|\left(E, E^{\prime}\right)$ satisfies the Lebesgue property if and only if $\tau$ does. The strong topology $\beta\left(E^{\prime}, E\right)$ on $E^{\prime}$ is always a locally solid topology. For proofs and more details see [1].

The following important result (essentially due to I. Amemiya) will be very influential to our work. Recall that a subset $A$ of a topological vector space is said to be precompact (or totally bounded) whenever for every neighborhood $V$ of zero, there exists a finite subset $X$ of $A$ such that $A \subseteq X+V$.

TheOREM 1.1. All Hausdorff Lebesgue topologies on a Riesz space induce the same topology on the order bounded subsets of the space.

In particular, all Hausdorff Lebesgue topologies on a Riesz space have the same order bounded precompact sets.

Proof. For the proof of the first part see [1, Th. 12.9, p. 87]. For the second part let $\xi$ and $\tau$ be two Hausdorff Lebesgue topologies on a Riesz space $E$, and let $A$ be an order bounded $\xi$-precompact subset of $E$. To complete the proof, we have to show that $A$ is also $\tau$-precompact.

To this end, let $W$ be a $\tau$-neighborhood of zero. Pick some $x \geqq 0$ with $A \subseteq[-x, x]$, and then choose a $\xi$-neighborhood $V$ of zero with $V \cap[-2 x, 2 x] \subseteq W \cap[-2 x, 2 x]$. Since $A$ is $\xi$-precompact, there exists a finite subset $X$ of $A$ with $A \subseteq X+V$. Now if $a \in A$, then choose some $b \in X$ with $a-b \in V$. In view of $-2 x \leqq a-b \leqq$ $2 x$, it follows that $a-b \in V \cap[-2 x, 2 x] \subseteq W \cap[-2 x, 2 x] \leqq W$. That is, $A \subseteq X+W$ holds, so that $A$ is $\tau$-precompact.

Another result that will be used extensively here is a beautiful duality theorem of A. Grothendieck. Recall that if $\left\langle E, E^{\prime}\right\rangle$ is a dual system and $\mathscr{A}$ is a collection of $\sigma\left(E, E^{\prime}\right)$-bounded subsets of $E$, then the $\mathscr{A}$-topology on $E^{\prime}$ is the locally convex topology (not 


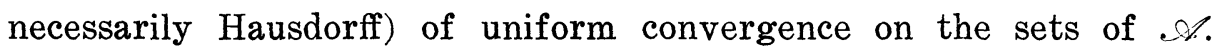
That is, the $\mathscr{A}$-topology is generated by the collection of seminorms $\left\{p_{A}: A \in \mathscr{A}\right\}$, where $p_{A}(f)=\sup \{|f(x)|: x \in A\}$ for each $f \in E^{\prime}$. Similarly, if $\mathscr{B}^{\prime}$ is a family of $\sigma\left(E^{\prime}, E\right)$-bounded subsets of $E^{\prime}$, then the $\mathscr{B}^{\prime}$-topology is the locally convex topology on $E$ (not necessarily Hausdorff) of uniform convergence on the sets of $\mathscr{B}$ '.

Recall also that if $T: E \rightarrow F$ is a weakly continuous operator corresponding to the dual systems $\left\langle E, E^{\prime}\right\rangle$ and $\left\langle F, F^{\prime}\right\rangle$, then the adjoint operator $T^{\prime}: F^{\prime} \rightarrow E^{\prime}$ is defined by

$$
\left\langle T^{\prime} f, x\right\rangle=\langle f, T x\rangle \text { for all } f \in F^{\prime} \text { and } x \in E \text {. }
$$

THEOREM 1.2 (Grothendieck). Let $\left\langle E, E^{\prime}\right\rangle$ and $\left\langle F, F^{\prime}\right\rangle$ be two dual systems and let $T: E \rightarrow F$ be a weakly continuous operator. If $\mathscr{A}$ is a collection of $\sigma\left(E, E^{\prime}\right)$-bounded subsets of $E$ and $\mathscr{B}{ }^{\prime}$ is a family of $\sigma\left(F^{\prime}, F\right)$-bounded subsets of $F^{\prime}$, then the following statements are equivalent:

1. $T(A)$ is precompact for the $\mathscr{B}^{\prime}$-topology for each $A \in \mathscr{A}$.

2. $T^{\prime}(B)$ is precompact for the $\mathscr{A}$-topology for each $B \in \mathscr{B}^{\prime}$.

For a proof of this theorem see [13, Th. 3, p. 51]. A special case of Theorem 1.2 useful to us here is the following: Let $T: E \rightarrow F$ be a weakly continuous operator between two locally convex spaces $(E, \xi)$ and $(F, \tau)$. Then $T$ maps $\xi$-bounded sets onto $\tau$-precompact sets if and only if $T^{\prime}$ maps equicontinuous subsets of $F^{\prime}$ onto $\beta\left(\boldsymbol{E}^{\prime}, \boldsymbol{E}\right)$-precompact sets.

We close this section with some well-known facts needed for our discussion. For a proof see [14, p. 85].

LEMMA 1.3. If $(E, \tau)$ is a locally convex space, then the following statements hold:

1. The topologies $\sigma\left(E, E^{\prime}\right)$ and $\tau$ agree on every $\tau$-precompact subset of $E$.

2. The topology $\sigma\left(E^{\prime}, E\right)$ and that of uniform convergence on the $\tau$-precompact subsets of $E$ agree on every $\tau$-equicontinuous subset of $E^{\prime}$.

2. Disjoint sequences and order precompactness. It is well known that many important properties of Riesz spaces can be expressed in terms of disjoint sequences. (A sequence $\left\{x_{n}\right\}$ in a Riesz space is said to be disjoint if $\left|x_{n}\right| \wedge\left|x_{m}\right|=0$ holds for all $n \neq m$.) In this work a few properties that are not well-known connected with disjoint sequences will be needed. They are of some independent interest, and they will be discussed in this section. 
We start with a technique, first used in [4], for constructing disjoint sequences.

Lemma 2.1. Let $E$ be a Riesz space and let $\left\{x_{n}\right\}$ be a sequence of $E^{+}$. If some $x \in E^{+}$satisfies $2^{-n} x_{n} \leqq x$ for all $n$, then the sequence $\left\{u_{n}\right\}$ defined by

$$
u_{n}=\left(x_{n+1}-4^{n} \sum_{i=1}^{n} x_{i}-2^{-n} x\right)^{+}
$$

is a disjoint sequence.

Proof. If $m>n \geqq 1$, then

$$
\begin{aligned}
0 \leqq 4^{-m} u_{m} & =\left(4^{-m} x_{m+1}-\sum_{i=1}^{m} x_{i}-2^{-8 m} x\right)^{+} \leqq\left(4^{-m} x_{m+1}-x_{n+1}\right)^{+} \\
& \leqq\left(2^{-n} 2^{-\langle m+1)} x_{m+1}-x_{n+1}\right)^{+} \leqq\left(2^{-n} x+4^{n} \sum_{i=1}^{n} x_{i}-x_{n+1}\right)^{+} \\
& =\left(x_{n+1}-4^{n} \sum_{i=1}^{n} x_{i}-2^{-n} x\right)^{-} \perp u_{n} .
\end{aligned}
$$

Our next lemma deals with disjoint sequences in the dual of a Riesz space.

Lemma 2.2. Let $(E, \tau)$ be a locally convex-solid Riesz space, and let $\left\{x_{n}\right\}$ be a disjoint sequence of $E$. If $\left\{f_{n}\right\}$ is a sequence of $E^{\prime}$, then there exists a disjoint sequence $\left\{g_{n}\right\}$ of $E^{\prime}$ with $\left|g_{n}\right| \leqq\left|f_{n}\right|$ and $g_{n}\left(x_{n}\right)=f_{n}\left(x_{n}\right)$ for all $n$.

Proof. Consider each $x_{n}$ embedded in $\left(E^{\prime}\right)_{\tilde{n}}$, and let $N_{n}=\left\{f \in E^{\prime}\right.$ : $\left.|f|\left(\left|x_{n}\right|\right)=0\right\}$ and $C_{n}=N_{n}^{d}$. Since $N_{n}$ is a band of $E^{\prime}$, we have $E^{\prime}=N_{n} \oplus C_{n}$. The relation $x_{n} \perp x_{m}$ for $n \neq m$ implies $C_{n} \perp C_{m}$ for $n \neq m\left[1, \mathrm{Th}\right.$. 3.10, p. 24]. If $g_{n}$ is the projection of $f_{n}$ onto $C_{n}$, then it is easy to verify that the sequence $\left\{g_{n}\right\}$ satisfies the desired properties.

The strong convergence of a sequence in the dual of a topologically complete locally convex-solid Riesz space is characterized in terms of disjoint sequences as follows. For Banach lattices the result has appeared in [5, Corollary 2.7, p. 297].

THEOREM 2.3. Let $(E, \tau)$ be a $\tau$-complete locally convex-solid Riesz space. Then for a sequence $\left\{f_{n}\right\}$ of $E^{\prime}$ the following statements are equivalent:

1. $\left\{f_{n}\right\}$ is $\beta\left(E^{\prime}, E\right)$-convergent to zero. 
2. $\left\{f_{n}\right\}$ is $|\sigma|\left(E^{\prime}, E\right)$-convergent to zero, and for each disjoint $\tau$-bounded sequence $\left\{x_{n}\right\}$ of $E^{+}$we have $\lim f_{n}\left(x_{n}\right)=0$.

Proof. $\quad(1) \Rightarrow(2)$ Since $|\sigma|\left(E^{\prime}, E\right) \subseteq \beta\left(E^{\prime}, E\right)$ holds and $\left\{f_{n}\right\}$ converges to zero uniformly on the $\tau$-bounded subsets of $E$, we see that (2) is immediate.

$(2) \Rightarrow(1)$ Assume by way of contradiction that a sequence $\left\{f_{n}\right\}$ of $E^{\prime}$ satisfies (2) but fails to satisfy (1). Then by passing to a subsequence it is easy to see that there exists a solid $\tau$-bounded subset $B$ of $E$ such that $f_{n} \notin B^{0}$ for each $n$.

Set $k_{1}=1$, and then choose $0 \leqq x_{1} \in B$ with $\left|f_{k_{1}}\right|\left(x_{1}\right) \geqq 1$. Next pick $k_{2}>k_{1}$ with $\left|f_{k_{2}}\right|\left(4 x_{1}\right)<1$ (such $k_{2}$ exists since $\lim \left|f_{n}\right|\left(x_{1}\right)=0$ ), and then choose $0 \leqq x_{2} \in B$ such that $\left|f_{k_{2}}\right|\left(x_{2}\right) \geqq 1$. Now by an inductive argument we can see that there exist $k_{1}<k_{2}<\cdots$ and a positive sequence $\left\{x_{n}\right\} \subseteq B$ such that the sequence $g_{n}=f_{k_{n}}$ satisfies

$$
\left|g_{n}\right|\left(x_{n}\right) \geqq 1 \text { and }\left|g_{n+1}\right|\left(4^{n} \sum_{i=1}^{n} x_{i}\right)<\frac{1}{n} \text { for all } n \text {. }
$$

Since $B$ is $\tau$-bounded, it is easy to see that $\left\{\sum_{i=1}^{n} 2^{-i} x_{i}\right\}$ is $\tau$-Cauchy. If $x$ is its $\tau$-limit in $E$, then clearly $0 \leqq 2^{-n} x_{n} \leqq x$ holds for each $n$. Thus, by Lemma 2.1, the sequence

$$
u_{n}=\left(x_{n+1}-4^{n} \sum_{i=1}^{n} x_{i}-2^{-n} x\right)^{+}
$$

is disjoint. In view of $\left\{u_{n}\right\} \subseteq B$, the sequence $\left\{u_{n}\right\}$ is also $\tau$-bounded. On the other hand, the inequalities

$$
\left|g_{n+1}\right|\left(u_{n}\right) \geqq\left|g_{n+1}\right|\left(x_{n+1}-4^{n} \sum_{i=1}^{n} x_{i}-2^{-n} x\right) \geqq 1-\frac{1}{n}-2^{-n}\left|g_{n+1}\right|(x)
$$

show that $\left|g_{n+1}\right|\left(u_{n}\right)>1 / 2$ must hold for all $n$ greater than some $m$. In view of $\left|g_{n+1}\right|\left(u_{n}\right)=\sup \left\{g_{n+1}(v):|v| \leqq u_{n}\right\}$, for each $n>m$ there exists some $\left|v_{n}\right| \leqq\left|u_{n}\right|$ with $g_{n+1}\left(v_{n}\right)>1 / 2$. Since $\left\{v_{n}^{+}\right\}$and $\left\{v_{n}^{-}\right\}$ are both $\tau$-bounded positive disjoint sequences, it follows from our hypothesis that $1 / 2<g_{n+1}\left(v_{n}\right)=g_{n+1}\left(v_{n}^{+}\right)-g_{n+1}\left(v_{n}^{-}\right) \rightarrow 0$, which is impossible. This contradiction completes the proof of the theorem.

The concept of order precompactness is needed for our discussion. This notion has appeared in a fragmented way in the works of many authors. M. Duhoux in [6] was the first who isolated and studied this property.

Start by observing that in a Riesz space $E$, if $S$ is a solid set and $x \geqq 0$, then

$$
[-x, x]+S=\left\{y \in E:(|y|-x)^{+} \in S\right\} .
$$


Indeed, if $y=a+b$ holds with $|a| \leqq x$ and $b \in S$, then $(|y|-x)^{+} \leqq$ $(|y|-|a|)^{+} \leqq|b|$, so that $(|y|-x)^{+} \in S$. On the other hand, if $(|y|-x)^{+} \in S$, then in view of $|y|=|y| \wedge x+(|y|-x)^{+} \in[-x, x]+S$ and the solidness of $[-x, x]+S$, we get $y \in[-x, x]+S$.

DeFinition 2.4. Let $(E, \tau)$ be a locally solid Riesz space, and let $A$ be a subset of $E$. Then $A$ is said to be order $\tau$-precompact if for each solid $\tau$-neighborhood $V$ of zero, there exists an element $x \geqq 0$ in the ideal generated by $A$ in $E$ such that $A \subseteq[-x, x]+V$ (or equivalently, such that $(|y|-x)^{+} \in V$ for all $\left.y \in A\right)$.

Precompactness implies order precompactness. (If $A \subseteq X+V$ holds with $X=\left\{x_{1}, \cdots, x_{n}\right\} \subseteq A$, then $x=\sum_{i=1}^{n}\left|x_{i}\right|$ belongs to the ideal generated by $A$ and satisfies $X \subseteq[-x, x]$, so that $A \subseteq[-x, x]+$ $V$ holds.) Since every order interval is $\tau$-bounded, it is easy to see that every order $\tau$-precompact set is necessarily $\tau$-bounded.

The next result characterizes in terms of disjoint sequences the order precompact sets in locally convex-solid Riesz spaces with the Lebesgue property.

Theorem 2.5. Let $(E, \tau)$ be a locally convex-solid Riesz space with the Lebesgue property. Then for a subset $A$ of $E$ the following statements are equivalent:

1. A is order $\tau$-precompact.

2. For every $\tau$-neighborhood $V$ of zero there exists some $y \in E^{+}$ such that $(|x|-y)^{+} \in V$ holds for all $x \in A$.

3. A is r-bounded, and every disjoint sequence in the solid hull of $A$ is $\tau$-convergent to zero.

Proof. $\quad(1) \Rightarrow(2) \quad$ Obvious.

(2) $\Rightarrow(3)$ Clearly, $A$ is $\tau$-bounded. Let $\left\{x_{n}\right\}$ be a disjoint sequence in the solid hull of $A$, and let $W$ and $V$ be two solid $\tau$-neighborhoods of zero such that $W+W \subseteq V$. Choose some $y \in E^{+}$with $(|x|-y)^{+} \in W$ for all $x \in A$; clearly, $\left(\left|x_{n}\right|-y\right)^{+} \in W$ holds for all $n$. Since $\tau$ is a Lebesgue topology and $\left\{\left|x_{n}\right| \wedge y\right\}$ is an order bounded disjoint sequence, there exists some $k$ with $\left|x_{n}\right| \wedge y \in W$ for all $n \geqq k$. Thus, for $n \geqq k$ we have

$$
\left|x_{n}\right|=\left(\left|x_{n}\right|-y\right)^{+}+\left|x_{n}\right| \wedge y \in W+W \cong V .
$$

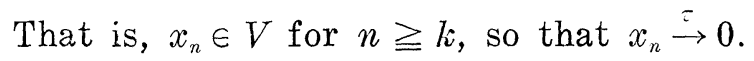

$(3) \Rightarrow(1)$ If $A$ is not order $\tau$-precompact, then there exists a solid $\tau$-neighborhood $V$ of zero such that for each $y$ in the ideal generated by $A$ we have $(|x|-y)^{+} \notin V$ for at least one $x \in A$. In 
particular, there exists a sequence $\left\{x_{n}\right\} \subseteq A$ with

$$
y_{n}=\left(\left|x_{n+1}\right|-4^{n} \sum_{i=1}^{n}\left|x_{i}\right|\right)^{+} \notin V \text { for all } n \text {. }
$$

The $\tau$-boundedness of $A$ implies that $\left\{\sum_{i=1}^{n} 2^{-i}\left|x_{i}\right|\right\}$ is a $\tau$-Cauchy sequence. Hence, there exists some $x$ in the topological completion $(\hat{E}, \hat{\tau})$ of $(E, \tau)$ with $\sum_{i=1}^{n} 2^{-i}\left|x_{i}\right| \stackrel{\hat{\tau}}{\rightarrow} x$. Clearly, $2^{-n}\left|x_{n}\right| \leqq x$ holds for all $n$.

Put $u_{n}=\left(\left|x_{n+1}\right|-4^{n} \sum_{i=1}^{n}\left|x_{i}\right|-2^{-n} x\right)^{+}$, and note that (by Lemma 2.1) $\left\{u_{n}\right\}$ is a disjoint sequence of $\hat{E}$. Moreover, we claim that $u_{n} \stackrel{\hat{\tau}}{\rightarrow} 0$. To see this, let $W$ and $U$ be two solid $\hat{\tau}$-neighborhoods of zero with $W+W \subseteq U$. Since $\hat{\tau}$ is a Lebesgue topology and $E$ is order dense in $\hat{E}$ [1, Thms 10.6 and 13.4], for each $n$ there exists some $v_{n} \in E$ with $0 \leqq v_{n} \leqq u_{n}$ and $u_{n}-v_{n} \in W$. Clearly, $\left\{v_{n}\right\}$ is a disjoint sequence lying in the solid hull of $A$ in $E$. Therefore, by our hypothesis $v_{n} \stackrel{\tau}{\rightarrow} 0$; pick some $k$ with $v_{n} \in W$ for $n>k$. Now if $n>k$, then $0 \leqq u_{n}=\left(u_{n}-v_{n}\right)+v_{n} \in W+W \subseteq U$ holds, so that $u_{n} \stackrel{\hat{\tau}}{\rightarrow} 0$.

Finally, the relation $0 \leqq y_{n} \leqq u_{n}+2^{-n} x \stackrel{\hat{\tau}}{\rightarrow} 0$, implies $y_{n} \stackrel{\tau}{\rightarrow} 0$, contrary to $y_{n} \notin V$ for each $n$. Therefore, $A$ is order $\tau$-precompact, and the proof of the theorem is finished.

A useful sufficient condition (in terms of disjoint sequences) for a set to be order precompact in topologically complete spaces is presented next.

THEOREM 2.6. Let $(E, \tau)$ be a $\tau$-complete locally convex-solid Riesz space, and let $A$ be a $\tau$-bounded subset of $E$. If every disjoint sequence in the solid hull of $A$ is $\tau$-convergent to zero, then $A$ is order $\tau$-precompact.

Proof. Repeat the arguments of the proof of the implication (3) $\Rightarrow(1)$ of Theorem 2.5.

The subsets of $E^{\prime}$ that are order $|\sigma|\left(E^{\prime}, E\right)$-precompact are referred to in [1] as order-equicontinuous sets. The next result characterizes the pre-Lebesgue property in terms of the concept of order precompactness.

THEOREM 2.7. For a locally convex-solid Riesz space $(E, \tau)$ the following statements are equivalent:

1. $\tau$ is a pre-Lebesgue topology.

2. Every disjoint equicontinuous sequence in $E^{\prime}$ is $\sigma\left(E^{\prime}, E\right)$ convergent to zero. 
3. Every equicontinuous subset of $E^{\prime}$ is order $|\sigma|\left(E^{\prime}, E\right)$-precompact.

Proof. $\quad(1) \Rightarrow(2)$ By replacing $(E, \tau)$ by its topological completion we can assume that $E$ is Dedekind complete and that $\tau$ is a Lebesgue topology [1, Th. 10.6]. Let $\left\{f_{n}\right\}$ be a positive disjoint equicontinuous sequence of $E^{\prime}$ and let $x \in E^{+}$. Set $N_{n}=\{y \in E$ : $\left.f_{n}(|y|)=0\right\}$ and $C_{n}=N_{n}^{d}$. Since $E^{\prime} \cong E_{n}^{\sim}$, each $N_{n}$ is a band, and so, $E=N_{n} \oplus C_{n}$ holds. If $x_{n}$ denotes the projection of $x$ onto $C_{n}$, then $\left\{x_{n}\right\}$ is an order bounded disjoint sequence [1, Th. 3.10], and so, $x_{n} \stackrel{\tau}{\rightarrow} 0$. Since $\left\{f_{n}\right\}$ is equicontinuous, it follows from $f_{n}(x)=f_{n}\left(x_{n}\right)$ that $\lim f_{n}(x)=0$, so that $f_{n} \stackrel{\sigma\left(E^{\prime}, E\right)}{\longrightarrow} 0$ holds.

$(2) \Rightarrow(1)$ Let $\left\{x_{n}\right\}$ be a disjoint sequence of $E$ satisfying $0 \leqq$ $x_{n} \leqq x$ for all $n$. We have to show that $x_{n} \stackrel{\tau}{\rightarrow} 0$ or, equivalently, that $\lim f_{n}\left(x_{n}\right)=0$ holds for an arbitrary equicontinuous sequence $\left\{f_{n}\right\}$ in $E^{\prime}$. By Lemma 2.2 we can assume that $\left\{f_{n}\right\}$ is disjoint. But then the inequality $\left|f_{n}\left(x_{n}\right)\right| \leqq\left|f_{n}\right|(x)$, coupled with our hypothesis, implies $\lim f_{n}\left(x_{n}\right)=0$.

$(2) \Leftrightarrow(3)$ The equivalence follows from Theorem 2.5 by observing that every equicontinuous subset of $E^{\prime}$ is $|\sigma|\left(E^{\prime}, E\right)$-bounded, and that $|\sigma|\left(E^{\prime}, E\right)$ is a Lebesgue topology on $E^{\prime}$.

The preceding theorem translated to the Banach lattice setting says that a Banach lattice has an order continuous norm if and only if every norm bounded disjoint sequence of continuous linear functionals is weakly convergent to zero. This special case was proven in [5, Cor. 2.8, p. 297].

The dual of Theorem 2.7 is stated next.

Theorem 2.8. For a locally convex-solid Riesz space $(E, \tau)$ the following statements are equivalent:

1. $\beta\left(E^{\prime}, E\right)$ is a Lebesgue topology.

2. Every disjoint sequence in the solid hull in $E^{\prime \prime}$ of a $\tau$ bounded subset of $E$ is $\sigma\left(E^{\prime \prime}, E^{\prime}\right)$-convergent to zero.

3. Every $\tau$-bounded subset of $E$ is order $|\sigma|\left(E, E^{\prime}\right)$-precompact.

Proof. Parallel the proof of Theorem 2.7, and use the fact that a subset of $E$ is order $|\sigma|\left(E, E^{\prime}\right)$-precompact if and only if it is order $|\sigma|\left(E^{\prime \prime}, E^{\prime}\right)$-precompact in $E^{\prime \prime}$.

In locally convex-solid Riesz spaces with the Lebesgue property, the concept of precompactness is related to order precompactness as follows. 
THEOREM 2.9. Let $(E, \tau)$ be a locally convex-solid Riesz space with the Lebesgue property. Then for a subset $A$ of $E$ the following statements are equivalent:

1. A is $\tau$-precompact.

2. A is $|\sigma|\left(E, E^{\prime}\right)$-precompact and order $\tau$-precompact.

3. A is $|\sigma|\left(E, E^{\prime}\right)$-precompact and every disjoint sequence in the solid hull of $A$ is $\tau$-convergent to zero.

Proof. (1) $\Rightarrow$ (2) Since $|\sigma|\left(E, E^{\prime}\right) \leqq \tau$ holds, $A$ is clearly $|\sigma|\left(E, E^{\prime}\right)-$ precompact. Also, we have seen before that precompactness implies order precompactness; see the discussion after Definition 2.4.

$(2) \Rightarrow(1)$ Let $V$ and $W$ be two solid $\tau$-neighborhoods of zero with $W+W \subseteq V$. Choose some $y \in E^{+}$with $(|x|-y)^{+} \in W$ for all $x \in A$, and hence, $\left(x^{+}-y\right)^{+} \in W$ for each $x \in A$. The relation $x^{+}=$ $\left(x^{+}-y\right)^{+}+x^{+} \wedge y$ shows that

$$
A^{+}=\left\{x^{+}: x \in A\right\} \subseteq W+A^{+} \wedge y,
$$

where $A^{+} \wedge y=\left\{x^{+} \wedge y: x \in A\right\}$.

The inequality $\left|x^{+} \wedge y-u^{+} \wedge y\right| \leqq|x-u|$ shows that $A^{+} \wedge y$ is $|\sigma|\left(E, E^{\prime}\right)$-precompact. Since $A^{+} \wedge y$ is order bounded $\left(A^{+} \wedge y \leqq\right.$ $[0, y])$, and both $|\sigma|\left(E, E^{\prime}\right)$ and $\tau$ are (Hausdorff) Lebesgue topologies, it follows from Theorem 1.1 that $A^{+} \wedge y$ is also $\tau$-precompact. Thus, for some finite set $X$ we have $A^{+} \wedge y \subseteq X+W$, and therefore, from (*) we get $A^{+} \leqq W+X+W \leqq X+V$. That is, $A^{+}$is $\tau$ precompact.

Similarly, it can be shown that $A^{-}=\left\{x^{-}: x \in A\right\}$ is $\tau$-precompact. Since $A \subseteq A^{+}-A^{-}$holds, we see that $A$ must also be $\tau$-precompact. (2) $\Leftrightarrow(3)$ This is immediate from Theorem 2.5.

REMARK. The equivalence of (1) and (2) in the preceding theorem remains true if $\tau$ is assumed to be only a pre-Lebesgue topology.

Repeating almost verbatim the arguments of the preceding proof, we can establish the following "dual" result of Theorem 2.9.

THEOREM 2.10. Let $(E, \tau)$ be a locally convex-solid Riesz space such that $\beta\left(E^{\prime}, E\right)$ is a Lebesgue topology. Then for a subset $A$ of $E^{\prime}$ the following statements are equivalent:

1. $A$ is $\beta\left(E^{\prime}, E\right)$-precompact.

2. A is $|\sigma|\left(E^{\prime}, E\right)$-precompact and order $\beta\left(E^{\prime}, E\right)$-precompact.

3. A is $|\sigma|\left(E^{\prime}, E\right)$-precompact, and every disjoint sequence in the solid hull of $A$ is $\beta\left(E^{\prime}, E\right)$-convergent to zero. 
3. Abstract kernel operators. Let $E$ and $F$ be Banach function spaces. Denote by $\left(E^{\prime} \otimes F\right)^{d d}$ the band generated by the (continuous) finite rank operators in $\mathscr{L}_{b}(E, F)$, the Dedekind complete Riesz space of all order bounded operators from $E$ into $F$. Since 1953, when $H$. Nakano in [12] was the first to recognize that there is a close relation between the operators in $\left(E^{\prime} \otimes F\right)^{d d}$ and the socalled kernel operators, there has been an extensive study of the compact operators in the band $\left(E^{\prime} \otimes F\right)^{d d}$; see [15, pp. 280-294]. As we have mentioned before, under certain conditions $\left(E^{\prime} \otimes F\right)^{d d}$ consists precisely of all kernel operators. Special cases of this property have been established by G. Ya. Lozanovskiǐ [8], R. J. Nagel and U. Schlotterbeck [11], and H. H. Schaefer [15]. When $E$ and $F$ are ideals of measurable functions, A. R. Schep [16] gave a rather general sufficient condition for $\left(E^{\prime} \otimes F\right)^{d d}$ to coincide with the band of all kernel operators. See also the historical remarks in [18].

In this section we consider $(E, \xi)$ and $(F, \tau)$ to be two (Hausdorff) locally convex-solid Riesz spaces with $F$ Dedekind complete. It is our purpose to study the compactness properties of the analogue of kernel operators in this setting.

If $E^{\prime} \otimes F$ denotes the vector space of all (continuous) finite rank operators, then $\left(E^{\prime} \otimes F\right)^{d d}$ will denote the band generated by $E^{\prime} \otimes F$ in $\mathscr{L}_{b}(E, F)$. We would like to call the members of $\left(E^{\prime} \otimes F\right)^{d d}$ kernel operators. However, since some kind of topological continuity is needed, we shall call every operator in $\left(E^{\prime} \otimes F\right)^{d d}$ that can be written as a difference of two positive weakly continuous operators, an (abstract) kernel operator. The collection of all kernel operators will be denoted by $\mathscr{K}(E, F)$.

REMARK. The reader may question why we require a kernel operator to be the difference of two positive weakly continuous operators. It is certainly true that particular results below also hold for other classes of operators in the band $\left(E^{\prime} \otimes F\right)^{d d}$. However, it will become apparent that some kind of continuity is needed.

Clearly, our definition of a kernel operator depends only upon the dual systems. In other words, all topologies consistent with the dual systems have the same kernel operators. This definition seems to contain the weakest conditions required to unify all the results.

The next result tells us that $\mathscr{K}(E, F)$ is an ideal of $\mathscr{L}_{b}(E, F)$, and its easy proof is left for the reader.

THEOREM 3.1. The kernel operators $\mathscr{K}(E, F)$ form an ideal of $\mathscr{L}_{b}(E, F)$ containing the (continuous) finite rank operators. 
In particular, note that if $\mathscr{K}(E, F)$ is a band, then $\mathscr{K}(E, F)=$ $\left(E^{\prime} \otimes F\right)^{d d}$ must hold. Also, note that if $T=T_{1}-T_{2}$ is a representation of a kernel operator as a difference of two positive weakly continuous operators, then the relation $T^{\prime}=T_{1}^{\prime}-T_{2}^{\prime}$ shows that the adjoint operator $T^{\prime}: F^{\prime} \rightarrow E^{\prime}$ is an order bounded operator. This observation will be used later.

The next result gives conditions under which all kernel operators share certain compactness properties. All the various characterizations of precompact kernel operators will depend upon this result.

THeOREM 3.2. Let $(E, \xi)$ and $(F, \tau)$ be two locally convex-solid Riesz spaces with $F$ Dedekind complete, and with $\tau$ and $\beta\left(E^{\prime}, E\right)$ Lebesgue topologies. Then for a kernel operator $T: E \rightarrow F$ the following statements hold:

1. $T[0, x]$ is $\tau$-precompact for each $x \in E^{+}$.

2. $T^{\prime}(A)$ is $|\sigma|\left(E^{\prime}, E\right)$-precompact for each equicontinuous subset $A$ of $F^{\prime}$.

3. $T^{\prime}[0, f]$ is $\beta\left(E^{\prime}, E\right)$-precompact for each $0 \leqq f \in F^{\prime}$.

4. $T(B)$ is $|\sigma|\left(F, F^{\prime}\right)$-precompact for each $\xi$-bounded subset $B$ of $E$.

Proof. (1) Let $x \in E^{+}$. Then $\mathscr{B}=\left\{T \in \mathscr{L}_{b}(E, F): T[0, x]\right.$ is $\tau$-precompact $\}$ is a band of $\mathscr{L}_{b}(E, F)$ [2, Th. 1.2]. Since $E^{\prime} \otimes F \subseteq$ $\mathscr{B}$ is obviously true, we infer easily that $\mathscr{K}(E, F) \subseteq\left(E^{\prime} \otimes F\right)^{d d} \subseteq$ $\mathscr{B}$ holds.

(2) Since $\tau$-convergence is the same as uniform convergence on the equicontinuous subsets of $F^{\prime}$, the conclusion follows from (1) by Theorem 1.2.

(3) Let $0 \leqq f \in F^{\prime}$. Then $[0, f]$ is an equicontinuous subset of $F^{\prime}$, and therefore, it follows from (2) that $T^{\prime}[0, f]$ is $|\sigma|\left(E^{\prime}, E\right)$ precompact. Since both $|\sigma|\left(E^{\prime}, E\right)$ and $\beta\left(E^{\prime}, E\right)$ are (Hausdorff) Lebesgue topologies on $E^{\prime}$ and $T^{\prime}[0, f]$ is order bounded (remember that $T^{\prime}$ is order bounded), it follows from Theorem 1.1 that $T^{\prime}[0, f]$ is $\beta\left(E^{\prime}, E\right)$-precompact.

(4) Apply Theorem 1.2 with $\mathscr{A}=\{B: B$ is a $\xi$-bounded subset of $E\}, \mathscr{B}^{\prime}=\left\{[0, f]: 0 \leqq f \in F^{\prime}\right\}$ and take into account (3).

Let us call a linear operator $T:(E, \xi) \rightarrow(F, \tau)$ between two locally convex spaces a precompact operator if it maps $\xi$-bounded sets onto $\tau$-precompact sets. (If $E$ and $F$ are Banach spaces, then the precompact operators are precisely the compact operators from $E$ into $F$.)

As mentioned before our main purpose is to characterize the 
precompact kernel operators. Our first result of this kind characterizes the precompact kernel operators in terms of approximation properties.

THEOREM 3.3. If $(E, \xi)$ and $(F, \tau)$ are as in Theorem 3.2, then for a kernel operator $T: E \rightarrow F$ the following statements are equivalent:

1. $T$ is a precompact operator.

2. For each $\xi$-bounded set $B$ and $\tau$-neighborhood $V$ of zero, there exists some $y \in F^{+}$such that

$$
(|T x|-y)^{+} \in V \text { for all } x \in B \text {. }
$$

3. For every $\xi$-bounded set $B$ and every $\tau$-neighborhood $V$ of zero, there exists some $y \in E^{+}$such that

$$
T\left[(|x|-y)^{+}\right] \in V \text { for all } x \in B .
$$

4. For each $\varepsilon>0$, each equicontinuous subset $A$ of $F^{\prime \prime}$, and each $\xi$-bounded subset $B$ of $E$, there exists some $0 \leqq g \in E^{\prime}$ such that

$$
\left|\left\langle\left(\left|T^{\prime} f\right|-g\right)^{+}, x\right\rangle\right|<\varepsilon \text { holds for all } f \in A \text { and } x \in B \text {. }
$$

5. For each $\varepsilon>0$, each equicontinuous subset $A$ of $F^{\prime}$, and each $\xi$-bounded subset $B$ of $E$, there exists some $0 \leqq g \in F^{\prime}$ such that

$$
\left|\left\langle T^{\prime}\left[(|f|-g)^{+}\right], x\right\rangle\right|<\varepsilon \text { holds for all } f \in A \text { and } x \in B \text {. }
$$

Proof. (1) $\Leftrightarrow(2) \quad$ By part (4) of Theorem 3.2, $T(B)$ is $|\sigma|\left(F, F^{\prime}\right)$ precompact for each $\xi$-bounded subset $B$ of $E$. Now the desired equivalence follows from Theorems 2.9 and 2.5.

$(1) \Leftrightarrow(4)$ By part (2) of Theorem 3.2, $T^{\prime}$ carries equicontinuous sets onto $|\sigma|\left(E^{\prime}, E\right)$-precompact sets. Our equivalence now follows by combining Theorems $2.10,1.2$, and 2.5.

(1) $\Rightarrow$ (3) Let $B \subseteq E$ be solid and $\xi$-bounded, and let $V$ be a solid $\tau$-neighborhood of zero. If (3) does not hold for the pair $B$ and $V$, then for each $y \in E^{+}$there exists some $x \in B$ with $T\left[(|x|-y)^{+}\right] \notin$ $V$. In particular, there exists a sequence $\left\{x_{n}\right\} \subseteq B$ with $T\left[\left(\left|x_{n+1}\right|-\right.\right.$ $\left.\left.4^{n} \sum_{i=1}^{n}\left|x_{i}\right|\right)^{+}\right] \notin V$ for all $n$. Now $\sum_{i=1}^{n} 2^{-i}\left|x_{i}\right| \uparrow$ holds in $E$ and $\left\{\sum_{i=1}^{n} 2^{-i}\left|x_{i}\right|\right\}$ is $\xi$-bounded. By [1, Th. 19.17, p. 131] there exists some $x \in E^{\prime \prime}$ such that $\sum_{i=1}^{n} 2^{-i}\left|x_{i}\right| \uparrow x$ holds in $E^{\prime \prime}$. Clearly, $2^{-n}\left|x_{n}\right| \leqq$ $x$ holds for all $n$.

Now the sequence $u_{n}=\left(\left|x_{n+1}\right|-4^{n} \sum_{i=1}^{n}\left|x_{i}\right|-2^{-n} x\right)^{+}$is (by Lemma 2.1) disjoint, and lies in the solid hull of $B$ in $E^{\prime \prime}$. Thus, by Theorem 2.8, $u_{n} \stackrel{\sigma\left(E^{\prime \prime}, E^{\prime}\right)}{\longrightarrow} 0$ holds. The relation 


$$
0 \leqq y_{n}=\left(\left|x_{n+1}\right|-4^{n} \sum_{i=1}^{n}\left|x_{i}\right|\right)^{+} \leqq u_{n}+2^{-n} x
$$

implies that $y_{n} \stackrel{\sigma\left(E, E^{\prime}\right)}{\longrightarrow} 0$. Hence, $T\left(y_{n}\right) \stackrel{\sigma\left(F, F^{\prime}\right)}{\longrightarrow} 0$. On the other hand, since $\left\{y_{n}\right\} \subseteq B$ holds and $T(B)$ is $\tau$-precompact (by our hypothesis), it follows from Lemma 1.3 that $T\left(y_{n}\right) \stackrel{\tau}{\rightarrow} 0$, contrary to $T\left(y_{n}\right) \notin$ $V$ for all $n$.

$(3) \Rightarrow(1)$ Let $B$ be a solid $\xi$-bounded subset of $E$. Set $A=$ $B \cap E^{+}$. In view of $B \subseteq A-A$, it is enough to show that $T(A)$ is $\tau$-precompact.

To this end, let $V$ be a solid $\tau$-neighborhood of zero. Pick some $0 \leqq y \in E$ with $T\left[(|x|-y)^{+}\right] \in V$ for all $x \in B$, and note that (by Theorem 3.2) $T[0, y]$ is $\tau$-precompact. But then the relation $T(x)=T\left[(x-y)^{+}\right]+T(x \wedge y)$ implies $T(A) \subseteq T[0, y]+V$, from which it follows that $T(A)$ is also $\tau$-precompact.

$(1) \Leftrightarrow(5)$ Parallel the proofs of the implications $(1) \Rightarrow(3)$ and $(3) \Rightarrow(1)$. For the implication $(1) \Rightarrow(5)$ take into account the following: Every $\tau$-equicontinuous sequence $\left\{f_{n}\right\} \subseteq F^{\prime}$ with $0 \leqq f_{n} \uparrow$ admits an accumulation point $f$ in $F^{\prime}$ for $\sigma\left(F^{\prime}, F\right)$ (since it is $\sigma\left(F^{\prime}, F\right)$ relatively compact), which implies that $f_{n} \uparrow f$ holds in $F^{\prime}$.

Characterizations of precompact kernel operators involving local continuity properties are presented next.

THEOREM 3.4. If $(E, \xi)$ and $(F, \tau)$ are as in Theorem 3.2, then for a kernel operator $T: E \rightarrow F$ the following statements are equivalent:

1. $T$ is a precompact operator.

2. The restriction of $T$ to each $\xi$-bounded subset of $E$ is continuous for the topologies $|\sigma|\left(E, E^{\prime}\right)$ on $E$ and $\tau$ on $F$.

3. The restriction of $T^{\prime}$ to each equicontinuous subset of $F^{\prime}$ is continuous for the topologies $|\sigma|\left(F^{\prime}, F\right)$ on $F^{\prime}$ and $\beta\left(E^{\prime}, E\right)$ on $E^{\prime}$.

Proof. $\quad(1) \Rightarrow(2)$ Let $\left\{x_{\alpha}\right\}$ be a $\xi$-bounded net with $x_{\alpha} \stackrel{|\sigma|\left(E, E^{\prime}\right)}{\longrightarrow} 0$. Clearly, $x_{\alpha} \stackrel{\sigma\left(E, E^{\prime}\right)}{\longrightarrow} 0$ holds, and by the weak continuity of $T$ we have $T x_{\alpha} \stackrel{\sigma\left(F, F^{\prime}\right)}{\longrightarrow} 0$. Now by our hypothesis $\left\{T x_{\alpha}\right\}$ is $\tau$-precompact, and so by Lemma $1.3, T x_{\alpha} \stackrel{\tau}{\rightarrow} 0$ holds, as required.

$(2) \Rightarrow(1) \quad$ It is enough to show that condition (3) of Theorem 3.3 holds. To this end, let $B$ be a solid $\xi$-bounded subset of $E$ and $V$ a $\tau$-neighborhood of zero. Since $0 \in B$, it follows from our hypothesis that there exists some $|\sigma|\left(E, E^{\prime}\right)$-neighborhood $U$ of zero such that $T(B \cap U) \subseteq V$. By statement (3) of Theorem 2.8, there exists 
some $y \in E^{+}$such that $(|x|-y)^{+} \in U$ for all $x \in B$. Since $(|x|-y)^{+} \epsilon$ $B$ also holds for each $x \in B$, it follows that $T\left[(|x|-y)^{+}\right] \in V$ for each $x \in B$, as required.

$(1) \Longrightarrow(3)$ Let $f_{\alpha} \stackrel{|\sigma|\left(F^{\prime}, F\right)}{\longrightarrow} 0$ in an equicontinuous subset of $F^{\prime}$. Since $f_{\alpha} \stackrel{\sigma\left(F^{\prime}, F_{1}\right)}{\longrightarrow} 0$ and since every $T(B)$ is $\tau$-precompact when $B$ is $\xi$-bounded, it follows from Lemma 1.3 that $f_{\alpha} \rightarrow 0$ uniformly on the set $T(B)$. That is, $T^{\prime} f_{\alpha} \stackrel{\beta\left(E^{\prime}, E\right)}{\longrightarrow} 0$.

$(3) \Rightarrow(1) \quad$ Repeat the proof of the implication $(2) \Rightarrow(1)$ using statement (3) of Theorem 2.7.

We now come to the characterizations of the precompact kernel operators in terms of disjoint sequences.

TheOREM 3.5. Let $(E, \xi)$ and $(F, \tau)$ be as in Theorem 3.2. Then for a kernel operator $T: E \rightarrow F$ the following statements are equivalent:

1. $T$ is a precompact operator.

2. If $B$ is a $\xi$-bounded set, then every disjoint sequence in the solid hull of $T(B)$ is $\tau$-convergent to zero.

3. Every disjoint equicontinuous sequence $\left\{f_{n}\right\} \subseteq F^{\prime}$ satisfies $T^{\prime} f_{n} \stackrel{\beta\left(E^{\prime}, E\right)}{\longrightarrow} 0$.

4. If $A$ is an equicontinuous subset of $F^{\prime}$, then every disjoint sequence in the solid hull of $T^{\prime}(A)$ is $\beta\left(E^{\prime}, E\right)$-convergent to zero.

Proof. $\quad(1) \Leftrightarrow(2) \quad$ By part (4) of Theorem 3.2, T(B) is $|\sigma|\left(F, F^{\prime}\right)$ precompact for each $\xi$-bounded subset $B$ of $E$. The equivalence of (1) and (2) now follows immediately from Theorem 2.9.

$(1) \Rightarrow(3)$ Let $\left\{f_{n}\right\}$ be a disjoint equicontinuous sequence in $F^{\prime}$. By Theorem 2.7, $f_{n} \stackrel{\sigma\left(F^{\prime}, F\right)}{\longrightarrow} 0$ holds. Since $\sigma\left(F^{\prime}, F\right)$ and the topology of uniform convergence on the $\tau$-precompact sets of $F$ agree on the equicontinuous subsets of $F^{\prime}$ (Lemma 1.3), it follows from this and our hypothesis that $f_{n} \rightarrow 0$ uniformly on the sets $T(B)$ with $B$ $\xi$-bounded. That is, $T^{\prime} f_{n} \stackrel{\beta\left(E^{\prime}, E\right)}{\longrightarrow} 0$ holds.

$(3) \Rightarrow(1)$ Let $B$ be a $\xi$-bounded subset of $E$. By part (4) of Theorem 3.2 we know that $T(B)$ is $|\sigma|\left(F, F^{\prime}\right)$-precompact. Thus, according to Theorem 2.9, we have to show that $y_{n} \stackrel{\tau}{\rightarrow} 0$ holds for each disjoint sequence $\left\{y_{n}\right\}$ in the solid hull of $T(B)$.

To this end, let $\left\{y_{n}\right\}$ be a disjoint sequence in the solid hull of $T(B)$. Choose $\left\{x_{n}\right\} \subseteq B$ with $\left|y_{n}\right| \leqq\left|T x_{n}\right|$ for all $n$, and let $\left\{f_{n}\right\}$ be a positive equicontinuous sequence in $F^{\prime}$. It remains to be shown that $\lim f_{n}\left(y_{n}\right)=0$. By Lemma 2.2 we can assume that $\left\{f_{n}\right\}$ is a disjoint sequence. Since 


$$
f_{n}\left(\left|T x_{n}\right|\right)=\sup \left\{\left|\left\langle g, T x_{n}\right\rangle\right|:|g| \leqq f_{n}\right\},
$$

for each $n$ there exists $\left|g_{n}\right| \leqq f_{n}$ such that

$(* *)$

$$
\begin{aligned}
\left|f_{n}\left(y_{n}\right)\right| & \leqq f_{n}\left(\left|T x_{n}\right|\right) \leqq\left|\left\langle g_{n}, T x_{n}\right\rangle\right|+\frac{1}{n} \\
& =\left|\left\langle T^{\prime} g_{n}, x_{n}\right\rangle\right|+\frac{1}{n} .
\end{aligned}
$$

Since $\left\{g_{n}\right\}$ is a disjoint equicontinuous sequence, it follows from our hypothesis that $T^{\prime} g_{n} \stackrel{\beta\left(E^{\prime}, E\right)}{\longrightarrow} 0$. From $\left(^{* *}\right)$ we now get immediately that $\lim f_{n}\left(y_{n}\right)=0$.

$(1) \Leftrightarrow(4)$ By statement (2) of Theorem 3.2, $T^{\prime}(A)$ is $|\sigma|\left(E^{\prime}, E\right)$ precompact for each equicontinuous subset $A$ of $F^{\prime}$. The desired equivalence follows now easily by combining Theorems 2.10 and 1.2.

If, in addition, $E$ is topologically complete, then we also have the following characterizations of the precompact kernel operators.

TheOREM 3.6. Let $(E, \xi)$ and $(F, \tau)$ be as in Theorem 3.2. If $E$ is $\xi$-complete, then for a kernel operator $T: E \rightarrow F$ the following statements are equivalent:

1. $T$ is a precompact operator.

2. For every disjoint $\xi$-bounded sequence $\left\{x_{n}\right\}$ in $E^{+}$we have $T\left(x_{n}\right) \stackrel{\tau}{\rightarrow} 0$.

3. For every disjoint $\xi$-bounded sequence $\left\{x_{n}\right\} \subseteq E^{+}$and for every disjoint equicontinuous sequence $\left\{f_{n}\right\} \subseteq F^{\prime}$ we have $\lim f_{n}\left(T x_{n}\right)=0$.

Proof. $\quad(1) \Rightarrow(2)$ Let $\left\{x_{n}\right\}$ be a disjoint $\xi$-bounded sequence in $E^{+}$. By Theorem 2.8, $x_{n} \stackrel{\sigma\left(E, E^{\prime}\right)}{\longrightarrow} 0$ holds, and so, $T\left(x_{n}\right) \stackrel{\sigma\left(F, F^{\prime}\right)}{\longrightarrow} 0$. Since $\left\{T\left(x_{n}\right)\right\}$ is $\tau$-precompact, it follows from Lemma 1.3 that $T\left(x_{n}\right) \stackrel{\tau}{\rightarrow} 0$.

(2) $\Rightarrow(3)$ Let $\left\{x_{n}\right\}$ be a $\xi$-bounded disjoint sequence of $E^{+}$, and let $\left\{f_{n}\right\}$ be a disjoint equicontinuous sequence in $F^{\prime}$. Then $\left\{T\left(x_{n}\right)\right\}$ converges to zero uniformly on $\left\{f_{n}\right\}$. In particular, $\lim f_{n}\left(T x_{n}\right)=0$ holds.

$(3) \Rightarrow(1) \quad$ It is enough to verify that statement (3) of Theorem 3.5 holds.

To this end, let $\left\{f_{n}\right\}$ be a disjoint equicontinuous sequence in $F^{\prime}$. Since $\tau$ is a Lebesgue topology, it follows from Theorem 2.7 that $f_{n} \stackrel{|\sigma|\left(F^{\prime}, F\right)}{\longrightarrow} 0$. From this, and the fact that $T$ can be written as a difference of two positive weakly continuous operators, it follows easily that $T^{\prime} f_{n} \stackrel{|\sigma|\left(E^{\prime}, E\right)}{\longrightarrow} 0$ holds. Therefore, by our hypothesis and 
Theorem 2.3 we see that $T^{\prime} f_{n} \stackrel{\beta\left(E^{\prime}, E\right)}{\longrightarrow} 0$ holds, and the proof of the theorem is finished.

REMARK. In the Banach lattice setting, Theorems 3.5 and 3.6 reduce to results that are contained in Thms 4.4, 5.2, 5.3, and 5.5 of [5].

Suppose $(E, \xi)$ and $(F, \tau)$ are two topological vector spaces. Let $\mathfrak{S}$ denote the collection of all $\xi$-bounded subsets of $E$ and let $\mathscr{N}$ be the collection of all $\tau$-neighborhoods of zero. Also, consider the vector space $\mathscr{L}(E, F)$ of all weakly continuous linear operators from $E$ into $F$. For each $B \in \mathfrak{S}$ and $V \in \mathscr{N}$ set

$$
U(B, V)=\{T \in \mathscr{L}(E, F): T(B) \subseteq V\} .
$$

It is easy to see that the family $\{U(B, V): B \in \mathcal{S}$ and $V \in \mathscr{N}\}$ forms a neighborhood basis at zero for a (Hausdorff) linear topology on $\mathscr{L}(E, F)$, called the S-topology; see [14, p. 79]. (Because of its obvious meaning, this topology is also referred to as the topology of uniform convergence on the bounded subsets of $E$.) We will always consider $\mathscr{L}(E, F)$ equipped with the $\subseteq$-topology. Note that if $E$ and $F$ are Banach spaces, then the S-topology on $\mathscr{L}(E, F)$ is simply the norm operator topology.

The rest of this section is devoted to characterizations of the precompact kernel operators in terms of projections. In order to ensure a sufficient number of projections we will assume that both spaces are Dedekind complete (this assumption can be weakened slightly). If $\left\{P_{n}\right\}$ is a sequence of band projections on a Riesz space $E$, then the symbol $P_{n} \downarrow 0$ in $E$ will mean that $P_{n+1} \leqq P_{n}$ holds for all $n$, and that $P_{n} x \downarrow 0$ for each $x \in E^{+}$.

Lemma 3.7. Let $E$ be a Dedekind complete Riesz space, and let $\left\{x_{n}\right\}$ be a disjoint sequence of $E$. Then there exists a sequence of band projection $\left\{P_{n}\right\}$ with $P_{n} \downarrow 0$ in $E$ and $P_{n}\left(x_{n}\right)=x_{n}$ for each $n$.

Proof. Let $P_{n}$ be the projection onto the band $B_{n}=\left\{x_{k}: k \geqq n\right\}^{d d}$. We claim that $\left\{P_{n}\right\}$ satisfies the desired properties. Indeed, note first that $P_{n} \downarrow$ and $P_{n}\left(x_{n}\right)=x_{n}$ for each $n$. On the other hand, if $0 \leqq y \leqq P_{n} x$ holds for all $n$, then it is easy to see that $y \perp x_{n}$ holds for all $n$. Therefore, $y \in B_{n} \cap B_{n}^{d}=\{0\}$ for all $n$, so that $P_{n} x \downarrow 0$ holds for all $x \in E^{+}$.

We now come to the characterizations of the precompact kernel 
operators in terms of projections.

THEOREM 3.8. Let $(E, \xi)$ and $(F, \tau)$ be two Dedekind complete locally convex-solid Riesz spaces such that $E$ is $\xi$-complete and both $\beta\left(E^{\prime}, E\right)$ and $\tau$ are Lebesgue topologies. Then for a kernel operator $T: E \rightarrow F$ the following statements are equivalent:

1. $T$ is a precompact operator.

2. $P_{n} T \stackrel{\mathfrak{S}}{\rightarrow} 0$ whenever $P_{n} \downarrow 0$ in $F$.

3. $P_{n} T Q_{n} \stackrel{\mathfrak{S}}{\rightarrow} 0$ whenever $P_{n} \downarrow 0$ in $F$ and $Q_{n} \downarrow 0$ in $E$.

If in addition, $T$ is $\sigma$-order continuous, then the above are equivalent to:

4. $T Q_{n} \stackrel{\mathfrak{S}}{\rightarrow} 0$ whenever $Q_{n} \downarrow 0$ in $E$.

Proof. $\quad(1) \Rightarrow(2) \quad$ Let $B$ be a $\xi$-bounded subset of $E$, and let $V$ and $W$ be two solid $\tau$-neighborhoods of zero with $W+W \subseteq V$. Since (by hypothesis) $T(B)$ is $\tau$-precompact, $T(B)$ is also order $\tau$ precompact. Pick $y \in F^{+}$with $(|T x|-y)^{+} \in W$ for all $x \in B$. In view of $P_{n} y \downarrow 0$, there exists some $k$ with $P_{n} y \in W$ for $n>k$. Thus,

$$
\left|P_{n} T x\right| \leqq P_{n}|T x| \leqq(|T x|-y)^{+}+P_{n} y \in W+W \subseteq V
$$

holds for all $n>k$ and $x \in B$. That is, $P_{n} T \in U(B, V)$ holds for all $n>k$, so that $P_{n} T \stackrel{\mathfrak{S}}{\rightarrow} 0$.

$(2) \Rightarrow(3)$ Note that if $B$ is a solid set, then $Q_{n}(B) \subseteq B$, and so, $P_{n} T Q_{n}(B) \subseteq P_{n} T(B)$ holds. From this observation the implication follows easily.

$(3) \Rightarrow(1)$ Let $\left\{x_{n}\right\}$ be a positive disjoint $\xi$-bounded sequence in $E$, and let $\left\{f_{n}\right\}$ be a disjoint equicontinuous sequence in $F^{\prime}$. According to Theorem 3.6 we have to show that $\lim f_{n}\left(T x_{n}\right)=0$.

By Lemma 3.7, there exist projections $Q_{n} \downarrow 0$ in $E$ with $Q_{n}\left(x_{n}\right)=$ $x_{n}$, and projections $P_{n}^{\prime} \downarrow 0$ in $F^{\prime}$ with $P_{n}^{\prime}\left(f_{n}\right)=f_{n}$. Since $\tau$ is a Lebesgue topology, there exists a sequence of projections $\left\{P_{n}\right\}$ on $F$ such that $\left\langle f, P_{n} x\right\rangle=\left\langle P_{n}^{\prime} f, x\right\rangle$ holds for all $f \in F^{\prime}$ and $x \in F$. (The projection $P_{n}$ is the projection of $F$ onto $\left[\left(P_{n}^{\prime} F^{\prime}\right)^{d}\right]^{0}$; see $[1, \mathrm{Th} .19 .5$, p. 127].) Using the Lebesgueness of $\tau$ once more we can see that $P_{n} \downarrow 0$ in $F$ holds.

Let $B=\left\{x_{n}\right\}$ and let $V=\left\{f_{n}\right\}^{0}$. Clearly, $V$ is a $\tau$-neighborhood of zero. Thus, given $\varepsilon>0$ there exists some $k$ with $P_{n} T Q_{n}(B) \cong \varepsilon V$ for all $n>k$. Therefore,

$$
\begin{aligned}
\left|f_{n}\left(T x_{n}\right)\right| & =\left|\left\langle f_{n}, T x_{n}\right\rangle\right|=\left|\left\langle P_{n}^{\prime} f_{n}, T x_{n}\right\rangle\right| \\
& =\left|\left\langle f_{n}, P_{n} T Q_{n} x_{n}\right\rangle\right| \leqq \varepsilon
\end{aligned}
$$

holds for all $n>k$, so that $\lim f_{n}\left(T x_{n}\right)=0$. 
$(4) \Longrightarrow(3)$ This follows from $\left|P_{n} T Q_{n} x\right| \leqq\left|T Q_{n} x\right|$.

$(1) \Longrightarrow(4)$ Assume that $T$ is also $\sigma$-order continuous. It is enough to show that if $\left\{x_{n}\right\}$ is a $\xi$-bounded sequence of $E^{+}$, then $T Q_{n} x_{n} \stackrel{\tau}{\rightarrow} 0$.

To this end, note first that $\left\{T Q_{n} x_{n}\right\}$ is weakly convergent to zero. Indeed, to see this fix $0 \leqq f \in F^{\prime}$. Since $Q_{n} x \downarrow 0$ holds for each $x \in E^{+}$, the $\sigma$-order continuity of $T$ implies $|T| Q_{n} x \downarrow 0$ in $F$. Thus, by the order continuity of $f$ we get

$$
\left\langle Q_{n}^{\prime}|T|^{\prime} f, x\right\rangle=\left\langle f,|T| Q_{n} x\right\rangle \downarrow 0 \text { for each } x \in E^{+} .
$$

That is, $Q_{n}^{\prime}|T|^{\prime} f \downarrow 0$ holds in $E^{\prime}$. In particular, since $\beta\left(E^{\prime}, E\right)$ is a Lebesgue topology, $\left\{Q_{n}^{\prime}|T|^{\prime} f\right\}$ converges to zero uniformly on $\left\{x_{n}\right\}$. Hence, $\left|\left\langle f, T Q_{n} x_{n}\right\rangle\right| \leqq\left\langle f,|T| Q_{n} x_{n}\right\rangle=\left\langle Q_{n}^{\prime}|T|^{\prime} f, x_{n}\right\rangle$, shows that $\left\{T Q_{n} x_{n}\right\}$ converges weakly to zero in $F$.

Now since $\left\{T Q_{n} x_{n}\right\}$ is also a $\tau$-precompact set, it follows from

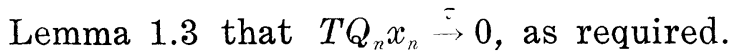

Theorem 3.8 has its roots in a paper of T. Andô [3]. For Orlicz spaces, he proved that statement (3) implies that a kernel operator is compact. Later W. A. J. Luxemburg and A. C. Zaanen [9], using measure-theoretical techniques, established the equivalence of (1) through (4) for kernel operators on Banach function spaces. Recall that by the Lebesgue dominated convengence theorem, a kernel operator on a Banach function space is automatically order continuous. P. van Eldik and J. J. Grobler in [7] with the additional hypothesis that $T$ is order continuous were able to establish the equivalence of (1) through (4) for the Banach lattice setting. However, we see from our proof that the equivalence of (1) through (3) does not rely on order continuity and that (4) requires only $\sigma$-order continuity.

4. Kernel operators on general spaces. The key to the preceding results rested upon the fact that $\beta\left(E^{\prime}, E\right)$ and $\tau$ were Lebesgue topologies. Now in this section our objective is to present compactness results on (abstract) kernel operators without assuming any Lebesgue properties. Obviously, to compensate for this loss, some additional assumptions on the operators must be made.

It is well known that a norm bounded subset of an abstract $L$ space is relatively weakly compact if and only if every disjoint sequence in its solid hull is norm convergent to zero; see, for example, [1, Th. 21.10, p. 153]. Evidently, this prompted P. MeyerNieberg [10] to give the following definition. 
DEFINITION 4.1. Let $T: E \rightarrow F$ be a linear operator between two locally convex-solid Riesz spaces $(E, \xi)$ and $(F, \tau)$. If for every $\xi$-bounded set $B$, each disjoint sequence in the solid hull of $T(B)$ is $\tau$-convergent to zero, then $T$ is called an L-weakly compact operator.

Observe that if $(F, \tau)$ is $\tau$-complete, then (by Theorem 2.6) each $L$-weakly compact operator maps $\xi$-bounded sets onto order $\tau$-precompact sets.

Lemma 4.2. Let $(E, \xi)$ and $(F, \tau)$ be two locally convex-solid Riesz spaces with $F$ Dedekind complete and $\tau$-complete. If $T: E \rightarrow F$ is a positive kernel operator that is L-weakly compact, then $T[0, x]$ is $\tau$-precompact for each $x \in E^{+}$.

Proof. Using the characterization of the pre-Lebesgue property in terms of disjoint sequences [1, Th. 10.1], it is easy to see that the $L$-weak compactness of $T$ implies that $\tau$ is a pre-Lebesgue topology on the ideal generated by $T(E)$ in $F$. Consequently, $\tau$ induces a Lebesgue topology on the $\tau$-closure $I$ of this ideal; see [1, Th. 10.6]. Note also that $I$ is an ideal of $F$.

Now choose a net $\left\{S_{\alpha}\right\}$ in the ideal generated by $E^{\prime} \otimes F$ in $\mathscr{L}_{b}(E, F)$ such that $0 \leqq S_{\alpha} \uparrow T$ holds. Since $S_{\alpha} x \uparrow T x$ (in $I$ or in $F$ ) holds for each $x \in E^{+}$, it follows from the Lebesgueness of $\tau$ on $I$ that $S_{\alpha} x \stackrel{\tau}{\rightarrow} T x$ holds for each $x \in E$. This in turn implies easily that $0 \leqq S_{\alpha}^{\prime} \uparrow T^{\prime}$ holds in $\mathscr{L}_{b}\left(F^{\prime}, E^{\prime}\right)$. Since each $S_{\alpha}^{\prime}$ is bounded by a finite rank operator (if $0 \leqq S_{\alpha} \leqq f \otimes u$, with $f \in E^{\prime}$ and $u \in F$, then $0 \leqq S_{\alpha}^{\prime} \leqq(f \otimes u)^{\prime}=u \otimes f$ holds, where $u$ is now considered as a functional on $F^{\prime}$ ), it follows from [2, Th. 1.2] that $T^{\prime}[0, g]$ is $|\sigma|\left(E^{\prime}, E\right)$-precompact for each $0 \leqq g \in F^{\prime}$. Therefore, by Theorem $1.2, T[0, x]$ is $|\sigma|\left(F, F^{\prime}\right)$-precompact, and hence, also $|\sigma|\left(I, I^{\prime}\right)$-precompact for each $x \in E^{+}$. Now to complete the proof, use statement (3) of Theorem 2.9.

Under certain conditions the squares of $L$-weakly compact operators are precompact operators.

THEOREM 4.3. Let $(F, \tau)$ be a $\tau$-complete locally convex-solid Riesz space that is Dedekind complete. If $T: F \rightarrow F$ is an L-weakly compact positive kernel operator, then $T^{2}$ is a precompact operator.

Proof. Let $B$ be a $\tau$-bounded subset of $F$, and let $V$ be a $|\sigma|\left(F, F^{\prime}\right)$-neighborhood of zero. Since $T$ is obviously continuous for $|\sigma|\left(F, F^{\prime}\right)$, there exists some $|\sigma|\left(F, F^{\prime}\right)$-neighborhood $W$ of zero 
with $T(W) \subseteq V$. Also, since $T$ is $L$-weakly compact, it follows from Theorem 2.6 that $T(B)$ is order $\tau$-precompact. Thus, there exists some $x \in F^{+}$such that $T(B) \subseteq[-x, x]+W$ holds. Therefore,

$$
T^{2}(B) \subseteq T[-x, x]+T(W) \subseteq T[-x, x]+V .
$$

Since (by Lemma 4.2) $T[-x, x]$ is $\tau$-precompact, it follows easily from the last relation that $T^{2}(B)$ is $|\sigma|\left(F, F^{\prime}\right)$-precompact.

Next note that if $I$ is the closure of the ideal generated by $T(F)$ in $F$, then $\tau$ restricted to $I$ is a Lebesgue topology. Thus, by statement (3) of Theorem 2.9, $T^{2}(B)$ is $\tau$-precompact, and the proof of the theorem is complete.

It should be noted that an operator that satisfies the hypotheses of Theorem 4.3 need not be a precompact operator. Examples of this kind are provided by the kernel operators of finite double norm on $L_{1}$-spaces; see $[17$, p. 322].

Finally, we close the paper with two sufficient conditions for a kernel operator to be a precompact operator.

TheOREM 4.4. Let $(E, \xi)$ and $(F, \tau)$ be two locally convex-solid Riesz spaces with $F$ Dedekind complete. Let $T: E \rightarrow F$ be a kernel operator such that for every $\xi$-bounded subset $B$ of $E$ and every $\tau$-neighborhood $V$ of zero in $F$, there exist $0 \leqq y \in E$ and $0 \leqq g \in F^{\prime}$ such that

$$
T\left[(x-y)^{+}\right] \in V \quad \text { and } \quad T^{\prime}\left[(f-g)^{+}\right] \in B^{0}
$$

hold for all $x \in B$ and $f \in V^{0}$. Then each of the following conditions implies that $T$ is a precompact operator:

1. $g$ can be chosen in $F_{n}^{\prime}=F^{\prime} \cap F_{n}^{\sim}$.

2. $T$ is positive and $F$ is $\tau$-complete.

Proof. (1) We first show that if $B$ is $\xi$-bounded, then $T(B)$ is $|\sigma|\left(F, F_{n}^{\prime}\right)$-precompact; we shall assume that $B$ is solid. Let $V$ and $W$ be two $|\sigma|\left(F, F_{n}^{\prime}\right)$-neighborhoods of zero such that $W+W \subseteq V$. Since they are also $\tau$-neighborhoods of zero, there exists some $0 \leqq$ $y \in E$ such that $T\left[(x-y)^{+}\right] \in W$ for all $x \in B$. Therefore, $T(B \cap E)^{+} \cong$ $T[0, y]+W$. On the other hand, since $|\sigma|\left(F, F_{n}^{\prime}\right)$ is a Lebesgue topology (not necessarily Hausdorff) on the Dedekind complete Riesz space $F$, it follows from [2, Th. 1.2] that $T[0, y]$ is $|\sigma|\left(F, F_{n}^{\prime}\right)$-precompact. Choose a finite set $X$ such that $T[0, y] \subseteq X+W$, and note that $T\left(B \cap E^{+}\right) \subseteq X+W+W \subseteq X+V$. That is, $T\left(B \cap E^{+}\right)$ is $|\sigma|\left(F, F_{n}^{\prime}\right)$-precompact, and since $B \subseteq B \cap E^{+}-B \cap E^{+}$, it follows that $T(B)$ is likewise $|\sigma|\left(F, F_{n}^{\prime}\right)$-precompact. Now by applying 
Theorem 1.2 we get that $T^{\prime}[0, g]$ is $\beta\left(E^{\prime}, E\right)$-precompact for each $0 \leqq g \in F_{n}^{\prime}$. Next we prove that if $A$ is a $\tau$-equicontinuous subset of $F^{\prime}$, then $T^{\prime}(A)$ is $\beta\left(E^{\prime}, E\right)$-precompact; we can assume that $A$ is solid. Let $U$ be a $\beta\left(E^{\prime}, E\right)$-neighborhood of zero. By our hypothesis, there exists $0 \leqq g \in F_{n}^{\prime}$ such that $T^{\prime}\left[(f-g)^{+}\right] \in U$ for all $f \in A$. Hence, $T^{\prime}\left(A \cap F_{+}^{\prime}\right) \subseteq T^{\prime}[0, g]+U$, and since $T^{\prime}[0, g]$ is $\beta\left(E^{\prime}, E\right)$ precompact, it follows easily that $T^{\prime}(A)$ is $\beta\left(E^{\prime}, E\right)$-precompact.

Finally, using Theorem 1.2 once more we get that $T$ is a precompact operator.

(2) Since for each solid $\xi$-bounded set $B$ and every $\tau$-neighborhood $V$ of zero, there exists $0 \leqq y \in E$ such that $T\left(B \cap E^{+}\right) \leqq$ $T[0, y]+V$, it suffices to prove that each $T[0, y]$ is $\tau$-precompact. By establishing that $T$ is $L$-weakly compact, this will follow from Lemma 4.2 .

To this end, let $\left\{z_{n}\right\}$ be a positive disjoint sequence in the solid hull of $T(B)$ for a solid $\xi$-bounded set $B$. We have to prove that $\boldsymbol{z}_{n} \stackrel{\tau}{\rightarrow} 0$. Let $A$ be a solid $\tau$-equicontinuous subset of $F^{\prime}$, and $\varepsilon>0$. For each $n$ choose some $0 \leqq x_{n} \in B$ such that $0 \leqq z_{n} \leqq T x_{n}$. By our hypothesis, there exist $0 \leqq u \in E$ and $0 \leqq g \in F^{\prime}$ such that

$$
T(x-u)^{+} \in \varepsilon A^{0} \quad \text { and } T^{\prime}(f-g)^{+} \in \varepsilon B^{0}
$$

holds for all $x \in B$ and $f \in A$. Then for each $f \in A$ we have

$$
\begin{aligned}
\left|f\left(z_{n}\right)\right| & \leqq|f|\left(z_{n}\right)=(|f|-g)^{+}\left(z_{n}\right)+|f| \wedge g\left(z_{n}\right) \\
& \leqq(|f|-g)^{+}\left(T x_{n}\right)+|f| \wedge g\left[\left(z_{n}-T u\right)^{+}+z_{n} \wedge T u\right] \\
& \leqq \varepsilon+|f| \wedge g\left[\left(T x_{n}-T u\right)^{+}\right]+g\left(z_{n} \wedge T u\right) \\
& \leqq \varepsilon+|f|\left[T\left(x_{n}-u\right)^{+}\right]+g\left(z_{n} \wedge T u\right) \leqq 2 \varepsilon+g\left(z_{n} \wedge T u\right) .
\end{aligned}
$$

Since the sequence $\left\{z_{n} \wedge T u\right\}$ is an order bounded disjoint sequence, it follows that $\lim g\left(z_{n} \wedge T u\right)=0$. Thus, $\left\{z_{n}\right\}$ converges to zero uniformly on the equicontinuous set $A$. Therefore, $z_{n} \stackrel{\tau}{\rightarrow} 0$ holds, and the proof of the theorem is complete.

\section{REFERENCES}

1. C. D. Aliprantis and O. Burkinshaw, Locally Solid Riesz Spaces, Academic Press, New York, 1978.

2. - Positive compact operators on Banach lattices, Math. Z., 174 (1980), 289-298.

3. T. Andô, On compactness of integral operators, Nederl. Akad. Wetensch. Proc. Ser.

A, 65 (1962), 235-239.

4. O. Burkinshaw and P. Dodds, Disjoint sequences, compactness, and semireflexivity in locally convex Riesz spaces, Illinois J. Math., 21 (1977), 759-775.

5. P. Dodds and D. H. Fremlin, Compact operators in Banach lattices, Israel J. Math., 34 (1979), 287-320. 
6. M. Duhoux, Order precompactness in topological Riesz spaces, J. London Math. Soc., (2), 23 (1981), 509-522.

7. P. van Eldik and J. J. Grobler, Lebesgue-type convergence theorems in Banach lattices with applications to compact operators, Nederl. Akad. Wetensch. Proc. Ser. A, 82 (1979), 425-437.

8. G. Ya. Lozanovskiì, On almost integral operators in KB-spaces, Vestnik Leningrad Univ. Mat. Mech. Astronomy, 7 (1966), 35-44.

9. W. A. J. Luxemburg and A. C. Zaanen, Compactness of integral operators in Banach function spaces, Math. Ann., 149 (1963), 150-180.

10. P. Meyer-Nieberg, Über klassen schwach kompakter operatoren in Banachverbänden, Math. Z., 138 (1974), 145-159.

11. R. J. Nagel and U. Schlotterbeck, Integraldarstellung regulärer operatoren auf Banachverbänden, Math. Z., 127 (1972), 293-300.

12. H. Nakano, Product spaces of semi-ordered linear spaces, J. Fac. Sci. Hokkaido Univ. Ser. I, 12 (1953), 163-210.

13. A. P. Robertson and W. Robertson, Topological Vector Spaces, 2nd Ed., Cambridge University Press, London, 1973.

14. H. H. Schaefer, Topological Vector Spaces, 3rd Printing, Springer-Verlag, Heidelberg, 1971.

15. - Banach Lattices and Positive Operators, Springer-Verlag, Heidelberg, 1974.

16. A. R. Schep, Kernel operators, Nederl. Akad. Wetensch. Proc. Ser. A, 82 (1979), $39-53$.

17. A. C. Zaanen, Linear Analysis, North-Holland, Amsterdam, 1960.

18. - Kernel operators. In: P. L. Butzer and B. Sz.-Nagy (Eds), Linear Spaces and Approximation, pp. 23-31, Birkhäuser Verlag Basel, 1978.

Received October 13, 1980 and in revised form May 19, 1981.

Department of Mathematical Sciences

INDIANA UNIVERSITY-PURDUE UNIVERSITY at INDIANAPOLIS

INDIANAPOLIS, IN 46205

U.S.A.

AND

Institut de Mathematique PURe et Appliquée

UNIVERSitÉ Catholique DE LOUVAIN

B-1348 LouvaIN-LA-NeUve

BELGTQUE 


\section{PACIFIC JOURNAL OF MATHEMATICS}

\section{EDITORS}

DONALD BABBITT (Managing Editor)

University of California

Los Angeles, California 90024

Hugo RossI

University of Utah

Salt Lake City, UT 84112

C. C. MOORE and ARTHUR Agus

University of California

Berkeley, CA 94720

\section{J. DUGUNDJI}

Department of Mathematics University of Southern California Los Angeles, California 90007

R. FinN and J. MILGRAM Stanford University Stanford, California 94305

\section{ASSOCIATE EDITORS}
R. ARNES
E. F. BeCKENBACH
B. H. Neumann
F. WOLF
K. YosHIDA

\section{SUPPORTING INSTITUTIONS}

UNIVERSITY OF ARIZONA

UNIVERSITY OF BRITISH COLUMBIA

CALIFORNIA INSTITUTE OF TECHNOLOGY

UNIVERSITY OF CALIFORNIA

MONTANA STATE UNIVERSITY

UNIVERSITY OF NEVADA, RENO

NEW MEXICO STATE UNIVERSITY

OREGON STATE UNIVERSITY
UNIVERSITY OF OREGON UNIVERSITY OF SOUTHERN CALIFORNIA STANFORD UNIVERSITY UNIVERSITY OF HAWAII UNIVERSITY OF TOKYO UNIVERSITY OF UTAH WASHINGTON STATE UNIVERSITY UNIVERSITY OF WASHINGTON 


\section{Pacific Journal of Mathematics}

Vol. 100, No. $1 \quad$ September, 1982

Charalambos D. Aliprantis, Owen Sidney Burkinshaw and M. Duhoux,

Compactness properties of abstract kernel operators $\ldots \ldots \ldots \ldots \ldots \ldots 1$

Roger C. Alperin, Locally compact groups acting on trees .............23

Robert F. Brown, Real homology of Lie group homomorphisms ......... 33

Karen Chase, Maximal groups in sandwich semigroups of binary relations . . 43

W. Wistar (William) Comfort and T. Soundararajan, Pseudocompact

group topologies and totally dense subgroups $\ldots \ldots \ldots \ldots \ldots \ldots \ldots 61$

M. Ferri and C. Gagliardi, Crystallisation moves $\ldots \ldots \ldots \ldots \ldots \ldots \ldots$

Kenneth R. Goodearl, Directly finite aleph-nought-continuous regular

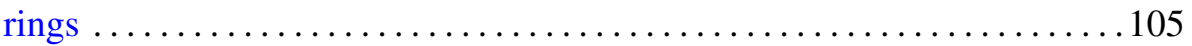

Edward Lewis Green, On the representation theory of rings in matrix

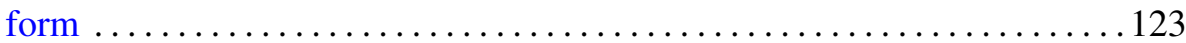

Walter Hengartner and Glenn E. Schober, Interpolation, continuation, and

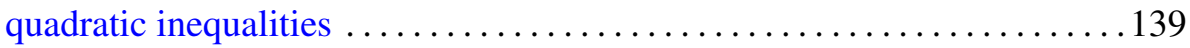

Kenneth Kunen and Haskell Paul Rosenthal, Martingale proofs of some geometrical results in Banach space theory $\ldots \ldots \ldots \ldots \ldots \ldots \ldots \ldots \ldots \ldots$

Brian William McEnnis, Shifts on indefinite inner product spaces. II . . . . . 177

Roman Pol, Note on the spaces $P(S)$ of regular probability measures whose topology is determined by countable subsets $\ldots \ldots \ldots \ldots \ldots \ldots \ldots \ldots 185$

Joan Manuel Verdera Melenchón, Finitely generated projective extensions

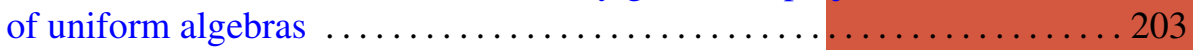

Cheng Ye You, Fixed point classes of a fiber map .................. 217 